\title{
Subjetividades y saberes docentes en el sistema educativo chileno: un análisis desde las concepciones de formadores de profesores, ${ }^{* * *}$
}

\author{
Teacher's knowledge and subjectivity in the Chilean Educational System: an analysis \\ from the teacher educator's conceptions
}

\section{Subjetividades e saberes docentes no sistema educacional chileno: uma análise desde as concepções de formadores de professores}

\author{
Leonora Reyes-Jedlicki, ${ }^{a}$ Christian Miranda J., ${ }^{b}$ Eduardo Santa Cruz G., ${ }^{a c}$ Rodrigo \\ Cornejo Ch., ${ }^{a d}$ Mauricio Núñez $R .,{ }^{a e}$ Ana Arévalo V.,${ }^{a f}$ Felipe Hidalgo K. ${ }^{a g}$ \\ aUniversidad de Chile. Correo electrónico: leoreyesj@gmail.com \\ bFACSO-Universidad de Chile. Correo electrónico: christian.miranda@u.uchile.cl \\ 'Correo electrónico: esantacruz1974@gmail.com. \\ dCorreo electrónico: rodrigo.cornejo@u.uchile.cl

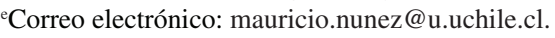 \\ ${ }^{\mathrm{f}}$ Correo electrónico: anarevalov@gmail.com \\ gCorreo electrónico: felipehidalgok@hotmail.com
}

\section{RESUMEN}

El presente estudio analiza las concepciones que los formadores de profesores poseen sobre las subjetividades y saberes de los docentes del sistema escolar chileno. El diseño de investigación consideró la aplicación de entrevistas en profundidad a nueve formadores de profesores de una universidad estatal. La información resultante fue procesada utilizando la Grounded Theory. Los resultados relevan las concepciones de los formadores de profesores respecto del valor pedagógico del saber y la subjetividad docente. Estas concepciones se discuten con la investigación internacional. Se concluye enfatizando la necesidad de dotar de especificidad al trabajo docente de tal manera de aportar insumos para la transformación de la formación docente.

Palabras clave: subjetividad docente, concepciones, trabajo docente.

\begin{abstract}
This study examines conceptions of teacher educators about teacher subjectivity and knowledge. The research design considered the application of in-depth interviews with nine state university teacher educators. The resulting data was processed using the Grounded Theory. Results reveal conceptions of teacher educators regarding the pedagogical value of knowledge and teacher's subjectivity; these are discussed taking into account the international knowledge and research on teaching. This research concludes by emphasizing the need to provide specificity to teaching work in such a way to provide input for the transformation of teachers' education.
\end{abstract}

Key words: teacher subjectivity, conceptions, teaching work.

Este trabajo se enmarca en el desarrollo del Núcleo de Investigación "Subjetividad docente en Chile: voces y experiencias desde el profesorado del sistema escolar", Iniciativa Bicentenario JGM, Fondo de Investigación / Creación, INTERD-2-3 y los proyectos FONDECYT No 1140744, No 1140827 y ํo 11130244 financiados por la Comisión Nacional de Investigación Científica y Tecnológica.

** Agradecemos la colaboración de Jenny Assaél, Jacqueline Gysling, Patricia Soto y Pablo Valdivieso. 


\section{RESUMO}

Analisam-se as concepções que os formadores de professores têm sobre as subjetividades e saberes dos docentes do sistema escolar chileno. O projeto de pesquisa considerou a aplicação de entrevistas em profundidade a nove formadores de professores de uma universidade estadual. A Teoria Fundamentada nos Dados (Grounded Theory) foi utilizada para processar os dados obtidos. Resultados revelam as concepções de formadores de professores a respeito do valor pedagógico do saber e da subjetividade docente. Tais concepções são confrontadas com a investigação internacional. Conclui enfatizando a necessidade de dotar de especificidade o trabalho docente de forma a contribuir para a transformação da formação de professores.

Palavras-chave: subjetividade docente, concepções, trabalho docente.

\section{INTRODUCCIÓN}

Durante la década del 90, los gobiernos democráticos intentaron corregir los impactos negativos de la reestructuración educacional producida durante la dictadura. En este contexto de inyección de recursos y reforma a importantes componentes del sistema escolar (currículo, financiamiento, uso de tecnología de la información, etc.), se promovieron acciones para el desarrollo profesional docente. La implementación del Programa de Fortalecimiento de la Formación Inicial Docente (FFID), hacia fines de 1990, fue una de esas acciones. Sin embargo, a mediados de la década siguiente, se evaluaron como moderados sus efectos, relevando la falta de una política efectiva y consensuada con los diversos actores involucrados, entre ellos, los formadores de profesores (Ávalos, 2005).

A partir de entonces, y como parte de la ejecución del Programa FFID, se desplegaron acciones concretas relacionadas con el mejoramiento de la calidad de los aprendizajes docentes. A la conformación de una Comisión designada especialmente para ello (2005), se sumó la creación de un Consejo Asesor Presidencial para la Calidad de la Educación (2006). Este último, mediante la instauración del Programa Inicia (2008), se centró en la consecución de dos objetivos principales. Uno, producir mejoras en el currículo de la FID (tanto en el ámbito disciplinar como pedagógico), orientándolo hacia uno de carácter "mínimo obligatorio" y de "calidad" y, otro, realizar una evaluación diagnóstica de los conocimientos pedagógicos y disciplinarios alcanzados por los egresados de las carreras de pedagogía (Prueba Inicia), además de apoyar con financiamientos parciales los programas de la FID. Finalmente, solo la prueba diagnóstica se ejecutó, dejando de lado la creación de espacios para dialogar y tomar acuerdos con las casas de estudio sobre el currículo y sus necesidades.

En este escenario, el foco de mejoramiento institucional de la FID, hasta la actualidad, ha estado centrado en la evaluación de los egresados. En efecto, desde 2010 -retirado en 2012- ingresó al Parlamento un "Examen de Excelencia Profesional Docente y Asignación de Excelencia Pedagógica" con lineamientos generales para la Prueba Inicia y con especial énfasis en su carácter no habilitante para ejercer funciones docentes. Desde marzo de 2012 hasta la fecha, sin embargo, se inició una nueva discusión en torno al proyecto "Sistema de promoción y desarrollo profesional docente para el sector municipal" conocido como Carrera Profesional Docente. Las indicaciones sustitutivas presentadas durante agosto de 2013, si bien no la volvieron habilitante, la declararon obligatoria con uso público de resultados. Asimismo, se propuso un plan de incentivos individuales que, sustentado en el artículo 46 de la Ley General de Educación (2009), posibilita el ejercicio de la docencia en enseñanza media con el solo hecho de tener una licenciatura en cualquier disciplina. 
Dicho de otra forma, este tipo de proyectos trae consigo ciertas prácticas que no contribuyen a la mejora de los procesos de aprendizaje de los docentes. Regular el ingreso de estos últimos al sistema escolar subvencionado, particular y municipal, mediante la utilización de una prueba de ingreso que tendría mayor peso que otros mecanismos regulatorios, tales como, la acreditación de las carreras de pedagogía; no es la forma más adecuada de enfrentar los desafíos de la formación de los docentes.

Por último, cabe destacar que, con el cambio de gobierno, la FID reaparece en el centro del debate educativo y, por ello, se crea una comisión ("Plan Maestro") que tiene como fin construir las bases de una "Nueva Profesión Docente" (2014) para el país a través de un diálogo ciudadano abierto, transparente y sistemático. No obstante, hasta ahora solo se ha incorporado un centro universitario de formación inicial docente.

En el Chile actual, las políticas de formación de docentes se sustentan en los énfasis propuestos por los informes OCDE $(2004,2009)$. De ahí que la atención se direccione hacia la FID y hacia la creación de incentivos para el ingreso a carreras de pedagogía (Beca Vocación de Profesor), evaluación de su egreso (Prueba Inicia) y procesos de acreditación obligatoria (realizada por agencias privadas). Para el caso de estos últimos procesos, no se consideran los requeridos y recomendados espacios de encuentro institucionales de participación, discusión y acuerdo con los formadores de profesores.

Si bien la relación entre formadores de profesores y las políticas de la FID ha sido débil, la relación que se genera entre los primeros y los docentes del sistema escolar no está libre de problemas. La literatura refiere una "tensión epistemológica” entre los formadores de profesores y los docentes en ejercicio con respecto al desarrollo de la investigación y el conocimiento. En efecto, una de las formas en que se expresaría dicha tensión es el tipo de concepción que se tiene sobre la tarea de enseñar. El formador de profesores buscaría patrones que permitan teorizar y mejorar las políticas educativas en torno a dicha tarea, o bien explicar los fenómenos relativos a ella, y, por su parte, el docente de aula demandaría aquello que solucione los problemas cotidianos en relación a su práctica de enseñanza en los establecimientos escolares (Joram, 2007).

Colaboración y coherencia son los principios inherentes de los programas de reforma (Russell, McPherson y Martin, 2001). Sin embargo, se reconoce que alcanzar la colaboración deseada entre universidad y escuela, y por extensión entre formadores de profesores y docentes en ejercicio profesional, es un desafío difícil de cumplir.

El presente artículo sostiene que analizar y problematizar las concepciones de académicos y académicas que participan en programas de investigación y formación docente con respecto a los saberes y subjetividad de los profesores del sistema escolar chileno, puede contribuir a acortar las brechas evidenciadas entre la FID, las políticas públicas y realidad de los docentes. Estudiar las convergencias y divergencias presentes en los relatos de formadores de profesores posibilita la visibilización de la voz del formador de profesores como actor clave en la transformación y mejora de la formación docente ${ }^{1}$.

Desde esta perspectiva, el posicionamiento epistemológico de la investigación se sitúa en la construcción colaborativa del conocimiento sobre las concepciones de los formadores de profesores, asumiendo algunas premisas del enfoque hermenéutico comprensivo.

Como autores de este artículo decidimos no hacer uso del género gramatical de la lengua española en la redacción de este escrito con el objeto de hacer más ágil su lectura. 
Estudios Pedagógicos, vol. XL, Número Especial 1: 183-203, 2014

SUBJETIVIDADES Y SABERES DOCENTES EN EL SISTEMA EDUCATIVO CHILENO: UN ANÁLISIS DESDE LAS CONCEPCIONES DE FORMADORES DE PROFESORES

En definitiva, el presente estudio no solo es la construcción de una respuesta a la necesidad institucional de generar convergencias entre los distintos formadores de profesores que investigan y participan en programas de formación docente en la Universidad de Chile, sino también es una forma de impulsar esta área investigativa en el marco del plan de reposicionamiento público de la Universidad referida.

\section{ANTECEDENTES CONCEPTUALES}

En este apartado se aborda una discusión teórica sobre la formación docente y la subjetividad de éste en el marco de una revisión de los enfoques en uso, y, además, se delimitan las premisas relativas a las concepciones y el lugar que ocupan éstas en los formadores de profesores.

En la investigación internacional sobre el docente y su formación, el debate se aborda desde, al menos, tres enfoques: uno que concibe la profesionalización de la enseñanza y la formación del profesorado desde estándares orientados a la docencia efectiva, programas de currículo planificados y una pedagogía que vincule teoría y práctica centrada en logros escolares (Darling-Hanmond, Chung Wei y Andree, 2010); otro orientado a la liberalización de la formación docente mediante el desmantelamiento de monopolios formativos, y que propone metas centradas en el desarrollo de habilidades sociales de los estudiantes (CochranSmith y Fries, 2005); finalmente, un tercero cuyo énfasis está en el docente como sujeto de las propuestas de formación, mediante temáticas tales como: entornos de enseñanza, el profesional como persona, la vida profesional de los docentes, el sostenimiento del compromiso de los profesores y el efecto del liderazgo (Day y Guy, 2010).

Estos tres enfoques configuran concepciones y propuestas formativas enmarcadas en campos de tensión permanentes: la brecha entre teoría-práctica y el conflicto disciplinapedagogía como foco de la formación, entre otros. Por lo tanto, se revela la necesidad de ir desarrollando una convergencia sobre la importancia de focalizar la investigación en los discursos que sustentan la práctica formativa (Rivera, 2014).

Bajo este contexto, las conceptualizaciones de subjetividad docente abordan, de manera compleja, inquietudes de larga data en investigación educativa. En efecto, entre sus líneas de estudio se encuentra la dimensión humana como construcción social, histórica y personal. Ejemplo de ello son los estudios internacionales sobre la personalidad de los docentes (Wittrock, 1986); la relación entre los atributos personales de los docentes y las condiciones escolares (Theobald, 1990); los trabajos de vida cotidiana en las aulas (Jackson, 1990; Rockwell, 2009); las investigaciones sobre biografías docentes (Day, 2006; Goodson, 2003), las investigaciones sobre saberes docentes (Tardiff, 2006) y sobre la historicidad docente (Freire, 1994; Zemelman, 1997, 2010), entre otros. En el caso de Chile, se sitúan en esta perspectiva las investigaciones sobre competencias docentes (Miranda, 2004); saber pedagógico (Montecinos, 2007); creencias y actitudes de los docentes (Becerra, 2009; Gómez, 2012); salud y el malestar docente (Cornejo, 2009; UNESCO, 2005; Valdivia et al., 2003); y culturas e identidades docentes (Acuña et al., 2014; López de Maturana, 2005; Núñez, 2003; Reyes et al., 2010; Reyes, Toledo y Egaña, 2013; Sisto, 2012).

En el debate latinoamericano reciente sobre estas temáticas, la categoría subjetividad docente ha sido entendida como "aquellos aspectos del pensamiento, de la voluntad, de las emociones, de valores, del lenguaje, de los deseos y las prácticas de los docentes" 
(Mancebo, 2010: 2). Se trata de un abordaje interdisciplinario que intenta asir la complejidad de tal categoría, entendiendo que ésta se constituye como una zona de sentido que permite nombrar una dimensión de lo social difícil de pensar (González Rey, 2002). Una dimensión que se reconstruye de manera histórica y que se sitúa en una interfaz entre las dimensiones individuales y sociales, y que no aspira a la coherencia y unicidad con que el pensamiento moderno concibe a los sujetos en tanto entes "autocentrados, incondicionados, transparentes y absolutamente racionales" (Acanda, 2008: 2).

En la presente investigación, las concepciones se asumen como organizadores implícitos de los conceptos de naturaleza esencialmente cognitiva y que incluyen creencias, significados, proposiciones, reglas, imágenes mentales y preferencias que influyen en lo que perciben los sujetos y en los procesos de razonamiento que éstos realizan (Moreno y Azcárate, 2003). Estas son muchas veces inconscientes y pueden ser individuales o colectivas, configurando una identidad respecto de algún fenómeno u objeto (Pozo et al., 2006; Sánchez, 2012). Así, las concepciones son una forma efectiva de dar explicaciones a los hechos o ideas a través de un discurso de sentido común. Por ejemplo, para tener una concepción sobre el saber docente no es necesario que exista una teoría o conocimiento científico respecto de este saber. En este marco, es posible sostener que los formadores de profesores a lo largo de la historia se han constituido por sus formas de vida profesional, cultura escolar, relaciones, y todo esto se cruza con las concepciones que estos mismos tienen respecto de sí (Rivera, 2014).

De acuerdo con Alvarado (2006), el formador de profesores es el profesional que, siendo parte de un colectivo, media la construcción de conocimientos con otros actores para, en conjunto, aprender y comprender conceptos y prácticas propias del ejercicio profesional. En esta definición se considera a todo aquel que socializa un saber o contribuye a implementar proyectos educativos desde las diversas áreas del conocimiento. Además, son considerados formadores de profesores los especialistas que planean e implementan actividades, proyectos o programas de una determinada política educacional o quienes participan de líneas de investigación educacional que incorporan la formación de tesistas de pre y postgrado.

Desde esta perspectiva, es indiscutible que el concepto formador de profesores abarca una amplia dispersión semántica. Efectivamente, como ya se ha mencionado, los formadores de profesores presentan características distintas y ocupan diversos lugares en las escalas formales académicas y en los organigramas de las instituciones educativas. Por ejemplo, académicos que realizan docencia en cursos de pregrado (licenciaturas, pedagogías y habilitación pedagógica para profesionales técnicos de nivel superior), postgraduación (especialización, magíster o doctorado) o de extensión (cursos, seminarios, talleres, entre otros). Dentro de esta categoría también se considera a profesionales del área de las ciencias sociales, las humanidades y la salud y, asimismo, profesores del sistema escolar que colaboran en el desarrollo profesional de los docentes.

Sin perjuicio de lo anterior, entender al sujeto formador de profesores requiere de un conocimiento sobre quiénes son, cómo se forman, qué necesidades y demandas tienen, qué culturas políticas, administrativas, pedagógicas, académicas y sociales los configuran, entre otros aspectos (Alvarado, 2006). De igual modo, exige comprender que el actuar del formador de profesores presupone concepciones y experiencias que avanzan desde la transferencia de contenidos a la construcción colectiva de conocimiento. Castillo (2006) agrega que el sujeto que actúa en la formación de docentes debe poseer autonomía y 
capacidad crítica. A pesar de los alcances previos, Koster (2005) plantea que los formadores de profesores son un grupo muchas veces omitido, tanto por las políticas educativas de formación de profesores como por los centros escolares.

A modo de síntesis, podemos señalar que el formador de profesores está condicionado por el contexto y las múltiples formas en que se despliega su saber. No obstante estos avances, los estudios no profundizan en la complejidad e imprevisibilidad de la tarea formativa (Korthagen, 2004; Zeichner, 2004). En este sentido, los estudios que indaguen sobre las concepciones, experiencias y prácticas cotidianas de los formadores de profesores, representan aportes al fortalecimiento de la profesión docente y al estudio de los formadores de profesores, actores generalmente relegados en la investigación educativa nacional.

\section{METODOLOGÍA}

En este estudio se indagan las concepciones que los formadores de profesores tienen sobre los saberes y la subjetividad de los docentes del sistema escolar. Considerando el interés por conocer los significados construidos socialmente por los formadores de profesores, el diseño de investigación es de carácter cualitativo, ya que, como sostiene Wright Mills (1969), los problemas son los que prescriben el método y no a la inversa. En este caso, se consideró aprovechar en beneficio del estudio la experiencia como formadores de profesores de los propios miembros del equipo de investigación. Por esto se utilizó como material empírico sus concepciones sobre la subjetividad y los saberes docentes en el contexto de su desempeño.

Investigaciones de este tipo cuentan con un importante desarrollo en el campo de los estudios cualitativos, como es el caso de la investigación participativa, el self study (Bullough y Pinnegar, 2001; Loughran et al., 2004), la autobiografía (Haynes, 2006), el enfoque biográfico narrativo (Arévalo, 2010; Van Manen, 2003), la auto-etnografía (Blanco, 2012) o los autorrelatos (Cruz, Reyes y Cornejo, 2012).

La particularidad del diseño metodológico de esta investigación es que quienes la conducen se toman a sí mismos como objeto de estudio, obligándose a construir simultáneamente diferentes posiciones enunciativas y a desarrollar un ejercicio reflexivo constante, con el fin de mantener la rigurosidad analítica. La aplicación de una reflexividad fuerte (Harding, 1996), que proporciona la construcción de conocimientos situados, supone la participación comprometida de los propios sujetos del conocimiento en auscultar y evaluar la propia posición de partida y las relaciones en que éste se inscribe (García, 2003). A partir de este modo de ver y hacer la investigación, surgieron interrogantes elementales, que Arévalo (2010: 193) describió como: “desde dónde emprendo la investigación, es decir, quién soy al investigar, cómo me relaciono con aquello que deseo indagar y qué licencias me doy al hacerlo -tanto los compromisos como los abandonos que decido-".

En armonía con lo anterior, los supuestos sobre los cuales se estructuró esta investigación fueron: i) el estudio de las vidas individuales de los formadores y de su subjetividad puede aportar a la comprensión de los contextos, los grupos y los espacios sociales en los cuales estos sujetos habitan (Blanco, 2012); ii) la experiencia del propio equipo de investigación dado su posición estructural, y bajo condiciones metodológicas específicas, puede ser escrutada analíticamente e integrada en el corpus del estudio.

En el trabajo participaron nueve formadores de profesores que encabezan investigaciones relativas a trabajo, profesionalidad y sujeto docente. Estos forman parte, a su vez, de ocho 
Grupos de Investigación (GI) pertenecientes a dos unidades académicas de la Universidad de Chile². Todos ellos se encuentran participando del Núcleo de Investigación "Subjetividad docente en Chile: voces y experiencias desde el profesorado del sistema escolar". Además, provienen de disciplinas distintas, como la psicología, la antropología, la filosofía, la historia, la pedagogía y la sociología. A pesar de ello, las diferencias de enfoque que existen en el grupo, como se evidencia en el análisis, trascienden a aquellas derivadas del origen disciplinario de los investigadores. La mayor parte de los integrantes no había trabajado en conjunto con antelación, y su participación en este proyecto se realizó en virtud de la confluencia temática, y no necesariamente en relación a los enfoques o perspectivas específicas para estudiar el fenómeno del trabajo docente. En suma, la muestra considerada representa una pluralidad discursiva al interior de los contextos institucionales en los que ellos trabajan.

La técnica utilizada para recabar la información fue la entrevista en profundidad, elaborada para los fines de la presente investigación. A partir de un conjunto de temas comunes en las entrevistas, la pauta final de cada entrevista fue adaptada a la trayectoria, intereses y perspectivas específicas de cada GI. Esta tarea fue acompañada de la revisión de algunas de sus investigaciones relacionadas con el trabajo, profesión y sujeto docente. El instrumento abordó cuatro dimensiones: la primera, el conocimiento de la trayectoria del formador de profesores; la segunda, el concepto de subjetividad docente; la tercera, la caracterización del sujeto docente y los contextos donde se desempeña; la cuarta, las estrategias metodológicas escogidas para estudiar al sujeto docente. Las entrevistas fueron desarrolladas entre agosto y diciembre del año 2013 y, posteriormente, transcritas de forma literal.

La técnica de análisis utilizada fue la Grounded Theory (Strauss y Corbin, 2002). Mediante el método comparativo constante y utilizando el software Atlas Ti, los discursos fueron analizados en dos fases: i) exploración línea a línea de los discursos y ii) levantamiento de categorías abiertas o emergentes. En este artículo se presentan las categorías que tienen relación exclusivamente con la concepción de los formadores de profesores sobre los saberes y la subjetividad docente, así como sobre las condiciones y posibilidades para su desempeño en instituciones escolares en Chile. Durante el proceso de análisis, el equipo de trabajo abordó el problema de la validez del conocimiento generado mediante un ejercicio reflexivo recursivo y continuo. Mediante esta espiral de análisis colectivo se buscó controlar los posibles sesgos individuales en el proceso y poner en discusión las concepciones de los formadores de profesores. Como forma adicional de controlar problemas de validez, se procedió a cotejar las entrevistas de los formadores con sus propios trabajos publicados en distintos espacios.

\section{ANÁLISIS DE RESULTADOS}

En este apartado se describen y analizan los principales resultados derivados del estudio de campo, de acuerdo con las categorías emergentes. Se realiza un análisis exploratorio por sujeto, una comparación constante del grupo de participantes y una contrastación interpretativa del marco conceptual propuesto y la discusión que de ello se genera.

Los ejes temáticos de los distintos GI son: GI1) Sujeto histórico docente en Chile; GI2) Construcción de la profesionalidad docente; GI3) Rol del profesor jefe y la complejidad de los consejos de curso; GI4) Formación de profesores y concepciones de evaluación de aprendizajes; GI5) Impacto del desarrollo profesional de profesores en la enseñanza y el aprendizaje; GI6) Efectos de la Subvención Escolar Preferencial en el trabajo docente y la cultura escolar; GI7) Significaciones en el trabajo docente respecto de la convivencia y violencia escolar; y GI8) Subjetividad, salud laboral y condiciones de trabajo de los docentes. 


\subsection{CONCEPCIONES SOBRE LOS SABERES DOCENTES}

Los participantes del estudio sostienen que los profesores del sistema escolar poseen ciertos saberes que no tienen relación únicamente con lo aprendido en los espacios formativos institucionales ni con lo evaluado por la política pública, sino que también existen aquellos que se forman en los establecimientos educacionales y que responden, en muchos casos, a pautas culturales y a una distribución de roles instalados en la sociedad chilena. Desde esta perspectiva, esta asignación diferencial de funciones y espacios al interior de la institución escolar, sobre la base de ciertos atributos, deviene en un determinado saber y le significa a quien lo posee una específica posición de poder e influencia. Es así como el género, la antigüedad en la institución o el componente generacional, entre otros, configuran "lugares donde unos tienen más poder que otros" (FP-GI6) ${ }^{3}$. A continuación, un ejemplo en que se identifican los saberes vinculados a lo generacional, y la manera en que ello se relaciona con el ejercicio del poder en los establecimientos escolares:

(...) en [la] escuela (donde se realiza la investigación) es muy importante ser viejo, eso te da status, los viejos son los que tienen más poder. (...) todo eso va configurando lugares donde unos tienen más influencia, pueden hacer unas cosas y no otras (FP-GI6).

Algo similar ocurre con los saberes docentes asociados al ser hombre y ser mujer en el establecimiento escolar. Habrían algunas características vinculadas con un saber, históricamente construido, que expresan un modo de estar y de relacionarse con otros (autoridades, pares, familias, etc.) de manera diferenciada por parte de hombres y mujeres:

(...) el [profesor] de media es más masculino, y en el consejo de profesores hablan más, son mal oyentes un poco, porque van menos, tienen más regalías también, se les trata con más privilegios... Porque los de básica están más controlados que el de media. (...) Por otro lado, el profe hombre va y habla con el director de uno a uno: '¡Buena compadre! y ahí lo resolvemos entre nosotros', pero la mujer es como más 'Don', es como un trato: ‘ioiga!', y es más coqueteo (FP-GI6).

El conjunto de los formadores de profesores señala que existe un ámbito de saberes que está relacionado con conocer la propia institución escolar, comprender sus formas, rutinas, valores y modos de relacionarse. Este saber moverse en la institucionalidad y en la cultura escolar se construye estando en ella. Este sentido práctico, no necesariamente consciente, a su vez genera un determinado sentido común que permea la cotidianidad de la institución escolar. Por ende, el periodo de inserción en el establecimiento escolar constituye un momento de adaptación al discurso y prácticas escolares, complementando la formación inicial entregada en la educación superior. Uno de los formadores de profesores -quien ejerció la docencia en un mismo establecimiento durante seis años- complementa esta idea señalando:

...ese colegio se convirtió para mí en una escuela, una escuela profesional. Yo vengo del mundo de la práctica, entonces fue en el mundo de la práctica donde primero aprendo estilos, formas de trabajo e incorporo formas de racionalidad (FP2-GI2).

Como mecanismo de identificación, en el artículo se ha considerado adecuado identificar a los distintos formadores de profesores en función de los grupos de investigación (GI) a los que pertenecen. 
Desde el saber que construye el docente en la interacción con la escuela (que puede plantearse como "un saber moverse" en el contexto de la institución escolar) emerge la siguiente interrogante: ¿cómo, al alero de este aprendizaje situado, se constituye un saber que represente un modo de saber-pensar propio de los profesores? Una de las primeras claves la ofrece uno de los formadores: el saber docente sería una construcción subjetiva, que se estructura sobre "perspectivas, la lente con que miro las cosas, o sea mis conocimientos, mis creencias, mis experiencias previas" (FP2-GI2). La subjetividad docente participa en la construcción de saberes prácticos, los que van constituyéndose en experiencia vivida, en la historicidad del docente. Al respecto, un formador de profesores asegura que el saber docente es alimentado por un "pensamiento casuístico", construido a lo largo de la trayectoria del docente:

...el pensamiento de los profesores es inductivo, no significa que los profesores no puedan desarrollar un razonamiento más deductivo (...) pero dentro, en el mundo de la práctica, el pensamiento docente es inductivo por excelencia, y dentro del pensamiento inductivo fundamentalmente es analógico, se desarrolla por sí solo. (...) [De esta manera va adquiriendo] conciencia práctica (...) [que] es todo lo que los profesores son capaces de hacer, pero sin ser capaces de decir por qué lo hacen, y no porque no tengan la capacidad de hacerlo, sino que no lo hacen porque no es necesario (FP2-GI2).

La conciencia práctica constituiría un saber anterior al estado reflexivo, al que puede llegarse desarrollando ciertas condiciones institucionales o individuales. De esta manera, una característica importante de los saberes docentes en aula es que estos se constituirían a partir de la intuición, como un estado pre-reflexivo asociado a saberes que van desarrollándose a partir de las múltiples y complejas situaciones que enfrenta el docente en la cotidianeidad: responder un mandato, asociarse con otros para buscar una solución a un problema interno del establecimiento, saber callar o saber actuar según posiciones de género, de clase, de trayectoria, etc. En definitiva, es saber adaptarse a la cultura institucional.

En síntesis, en la visión de los formadores sobre qué es y cómo se constituye el saber docente se destaca el rasgo de saber situado, esto es construido en la esfera del contexto de la escuela, a instancia de lógicas institucionales, relacionales, de ejercicio del poder, de aspectos culturales, de los requerimientos de la práctica, desde donde se ponen en acción y desarrollan modos de actuar-pensar. Esta dinámica va configurando los saberes que construye el docente, más allá de las instancias de formación inicial y de lo que se espera como saber desde las políticas curriculares y evaluativas que recaen sobre los profesores.

\subsubsection{Saber y profesionalidad docente}

Desde la visión de uno de los formadores, saber y profesionalidad docente son nociones estrechamente vinculadas, pero distintas entre sí. Si la conciencia práctica es un nivel primario en el desarrollo de la profesionalidad docente, la conciencia discursiva implica un grado más intenso de conciencia, pues requiere la interpelación de un otro que promueva la interrogación y la posterior interpretación. Llegando a este punto, puede adquirir un nivel todavía más complejo, como lo describe un formador de profesores: "Yo propuse en mi tesis pasar a la conciencia enunciativa, porque estaba muy claro en mi mente que era necesario escribir para poder tomar conciencia" (FP2-GI2). 
Desde esta perspectiva, la profesionalidad docente se vincularía a tres niveles de conciencia: práctica, discursiva y enunciativa. Éstas estarían involucradas en la praxis y tendrían que ver con "una toma de conciencia sobre mis haceres (...) sobre lo que digo en la escuela y cómo lo digo" (FP2-GI2). Esta visión concuerda con la propuesta de Connelly y Clandinin (1994) acerca de la relación entre saber y la noción de desarrollo profesional docente. Este enfoque ofrece, a través de la metáfora de la "reconstrucción", una clave para comprender el modo en que un docente aprende: el relato continuo y constante de experiencias vividas que al recrearse en el acto de contarlas y re-contarlas generan saber, saber de experiencia, radicando en esto su potencial transformador.

Desde una visión distinta, la relación entre saber y profesionalidad se sostiene en la idea de que el soporte teórico, construido de modo previo e independiente de la experiencia del profesor, sustenta y posibilita la capacidad reflexiva y, por tanto, profesional del docente:

...les falta profesionalización, en el sentido que necesitan manejar más teoría explícita para lo que están haciendo, entonces (...) yo lo que percibía era que no sabían, no se manejaban teóricamente (FP-GI3).

La profesionalidad del docente, desde este enfoque, no se vincularía con la capacidad de teorizar o generar conocimiento por y desde sí, de parte del profesor, sino con la capacidad de utilizar la teoría disponible (gestada de un modo previo y externo al profesor). En este sentido, queda desestimada la posibilidad de entender al docente como un "intelectual transformador", en tanto no estaría provisto del recurso teórico para desplegar aquella capacidad reflexiva, posibilitadora de transformación:

... porque al profesor le gusta esa actividad práctica, entonces, este práctico reflexivo ha tenido más que nada de práctico, no sé si podrá dar el paso reflexivo (FP-GI3).

Así, los formadores de profesores transitan en una dualidad establecida entre dos polos: uno que concibe en los docentes capacidades reflexivas propias, y otro que propone que dichas capacidades se encuentran disminuidas por la falta de teoría.

Considerando otra arista del trabajo docente, una formadora de profesores sostiene que a éste se lo concibe en una tensión dada entre las exigencias del sistema y su inclinación hacia lo formativo. Ejemplo de ello sería el Sistema de Medición de la Calidad de la Educación (SIMCE) y su carácter instructivo: "[al docente] le van a decir: 'no, no haga eso, preocúpese de puro instruir', cuando ellos ven su principal función en lo valórico, en la educación valórica" (FP-GI3). Desde esta perspectiva, las exigencias de centrar la acción y saber docente en lo instruccional, propiciado por la lógica de las pruebas estandarizadas, desplazaría aquello que el profesorado valora como su espacio propio: "hasta hoy se repite que este espacio es súper importante, porque es el que le permite ser casi su vocación [que] va más por ese lado, por el formar" (FP-GI3). El énfasis puesto en la dimensión formativa puede ser entendido como una reacción a las políticas de estandarización y a las prácticas institucionales en el sistema escolar. Desde esta óptica, dicha dimensión constituiría el núcleo de la profesión docente, y por tanto su ámbito de saber más propio.

Según algunos formadores de profesores, la naturaleza relacional de la profesión docente, que implica dimensiones emocionales y valóricas, sería un rasgo culturalmente asociado con el rol femenino: 
(...) [Existe] el interés por el otro, por el niño, etc. Eso me permite concluir que para las profesoras, a lo mejor podría haber diferencias con los varones, pero para las profesoras este sería un espacio que ellas llaman de formación, donde se juegan los valores y no simplemente de educación e instrucción (...) (FP-GI3).

En síntesis, de acuerdo con lo planteado por los formadores de profesores, la profesión docente oscilaría entre la inclinación a lo formativo, correspondiente a los saberes entendidos como relacionales, complejos y contextualizados; y la presión por lo instruccional, cuyos saberes serían concebidos como contenidos dados y sujetos a la evaluación por la política pública. La idea de desarrollo de consciencia profesional docente pone de relieve las categorías de conciencia práctica, saberes generados en el propio contexto escolar y en la experiencia individual-colectiva del docente, así como también se pone de manifiesto en la visión de los entrevistados la dualidad: saber-profesional basado en capacidades reflexivas propias versus capacidades docentes disminuidas por la falta de teoría.

\subsection{SUJETO DOCENTE EN LA ESCUELA ACTUAL: ENTRE REPRODUCIR Y TRANSFORMAR}

Para los formadores de profesores, las funciones y sentidos de educar transitan por una dualidad configurada entre concebir al docente como un reproductor de conocimientos o un intelectual transformador. Desde sus perspectivas, los docentes reproductores son aquellos que cumplen con el logro de los objetivos de aprendizaje descritos en el currículo, sin reflexionar ni cuestionar su propio ejercicio pedagógico. Por el contrario, el tipo de prácticas transformadoras de un intelectual transformador son aquellas que derivan de un ejercicio reflexivo respecto a su quehacer pedagógico y consideran que este posee un rol de transformación social. Estas figuras corresponderían, desde la óptica de los formadores, a tipos ideales que un mismo docente puede ocupar en diferentes momentos de su práctica pedagógica cotidiana. Un ejemplo de esta dualidad se expresaría en:

(...) por un lado, [los docentes] respondemos estratégica e instrumentalmente a la función que tenemos por cumplir, en términos pedagógicos, didácticos, evaluativos, pero, por otro lado, somos intelectuales críticos ante la realidad, hay un malestar docente, hay una crítica permanente al sistema escolar de parte de los profesores. (FP-GI5).

La descripción que los formadores de profesores hacen sobre los docentes manifiesta que estos últimos emplean una serie de acciones que han denominado de diversas formas: "estrategias de sobrevivencia", "simulaciones pedagógicas", entre otras. Las características de estas prácticas docentes estarían asociadas a la variabilidad en las condiciones históricas del ejercicio de la profesión. Es así como en el contexto de las políticas de rendición de cuentas y responsabilización, el docente se sitúa en la disyuntiva de educar integralmente a los estudiantes o prepararlos para que rindan exitosamente las pruebas estandarizadas. Aquí, los formadores de profesores observan que los docentes están construyendo simulaciones pedagógicas, o lo que Taylor (2006) denomina coreografía del accountability. En este escenario, los protagonistas, simultáneamente, simulan modificar sus prácticas y quienes los evalúan simulan creer que estas transformaciones son genuinas.

Esta forma de actuación docente se puede entender como un modo de satisfacer las demandas de control de los evaluadores o, dicho de otro modo, como la necesidad de adaptarse a contextos institucionales complejos, configurados por las políticas de rendición de cuentas. 
Una forma de expresarlo es: "la política te encarna, te hace sujeto" (FP-GI6). Y en este impactar e introyectarse la política en el sujeto, este resuelve: “...no llega y acepta la política, algo hace ahí entre medio, y allí hay que ir mirando qué, pero claro, no es un mero receptor” (FP-GI6).

De acuerdo con lo que sostienen algunos formadores de profesores, en esta dinámica de "aceptar a medias" las políticas en la escuela, de llevar adelante el juego de la simulación, el docente está poniendo en marcha una resistencia que manifiesta una tensión entre las políticas y su propio saber. Los docentes habrían aprendido a resistir pasivamente, estar en "el doble juego de criticar la política, pero también de asimilarla", es la lógica del "simulacro", "pasar piola", "se invisibilizan, es como una especie de actitud y gesto corporal de que "no me vean"” (FP-GI6).

(...) aunque efectivamente digas que el 'profe es un actor', es un actor que, como gran parte de esta sociedad es mucho más pasivo como docente, como ciudadano, que antes, entonces las brechas de las resistencias son menores y son como más sutiles por decir algo, no son tan abiertas, y eso también es difícil entender. (FP-GI6).

En suma, esta concepción entiende la pasividad como resistencia, especialmente cuando la política exige actuar en direcciones en las que los docentes no se sienten representados. En ese sentido, la constitución de su subjetividad se produciría en la tensión entre cumplir el conjunto de funciones que el sistema escolar les demanda y desarrollar autónomamente su capacidad creadora y transformadora. Como señala una de las formadoras de profesores:

(...) [al profesor] se le pide desempeñar muchas cosas en los distintos momentos en la historia, funciones de adoctrinamiento, funciones de ciudadanización, funciones de moralización, de acompañar al Estado a establecer cierto orden, y no solo al comienzo, sino que siempre; y por otro lado, [está] toda esa voluntad subjetiva, creativa, la posibilidad de crear lo nuevo, lo original, lo distinto, pensando en que cada uno de sus educadores es un sujeto particular e irrepetible. (FP-GI1).

En esta tensión se encontraría en juego también el reconocimiento del docente en tanto sujeto con capacidad de acción y visión propias, caracterizado por esta dimensión subjetiva, potencialmente emancipadora:

(...) hay una especie de malestar, pero también una crítica, y que tiene que ver con ese componente subjetivo, que yo veo como esta idea de emancipación, esta lucha permanente de los profesores por ser reconocidos y a la vez lograr seguridad (...) pero están en esa tensión permanente de hacer lo que se tiene que hacer, porque se les dice lo que tienen que hacer, y hacer lo que quieren hacer desde su visión de mundo. (FP-GI5).

Desde otra perspectiva asociada a los formadores de profesores, se sostiene que la posibilidad de resistencia se encuentra profundamente constreñida por un marco institucional y una producción de subjetividad que ha sido permeada por el discurso neoliberal, el que suele ir en correspondencia con la matriz general que prima en la sociedad. Así, la pasividad y el aislamiento que se observaría en los establecimientos escolares, como resultado de los dispositivos de control y vigilancia, tendría un carácter conservador antes que transformador (FP-GI6).

Antes la resistencia era mucho más clara, había organización docente, se manifestaba la resistencia, había movimiento pedagógico, desde lo pedagógico había resistencias al 
currículum oficial (...) entonces cuesta mucho mirar en los espacios escolares, tu puedes ir afuera, ir a la escuela sindical y vas a verlo más porque son colectivos, pero esos colectivos son profes de una escuela por acá, otro por allá, no están articulados. Entonces, es un tejido que no está articulado, lo que permea es el control, la vigilancia, es la pasividad. (...) yo siento que oscilan, no es tan claro, o sea veces es 'tenemos que hacer esto', 'igual nos sirve' y otras veces es 'esto no tiene ningún sentido, solo nos están vigilando'. (FPGI6).

Entonces, para este segundo grupo de formadores de profesores, un rasgo característico de la subjetividad docente sería una creciente tendencia a la individualización y la pérdida de los referentes colectivos. En ese sentido, predominarían las respuestas individuales a los problemas colectivos -algo propio de la solución posmoderna al problema del riesgo, según Bauman (2000)- lo que se explicaría, en buena medida, por la debilidad de las organizaciones sindicales que los agrupan y la existencia de contextos laborales flexibles y altamente precarizados.

...quizás en el pasado hubo un SUTE [Sindicato Único de Trabajadores de la Educación] no sé qué, hubo, hay esperanzas de que hubo experiencias de colectivo (...) [hoy] no hay en la escuela delegado del Colegio de Profesores, se tratan de hacer asambleas y se caen y generan muchos conflictos, porque en la asambleas no se respetan los acuerdos de las otras asambleas, entonces se quiebra. Entonces, el pensarse como un sujeto un poquito más colectivo es así... y eso lo que significa es como la idea de la isla, de que tú estás aislado, y ahí está el aguantar fuerte, tienes que aguantar solo, porque es distinto aguantar yo, que aguantemos nosotros, porque ahí ya no es solamente aguantar, sino que puedes hacer otras cosas. (FP-GI6).

De acuerdo con esto, ciertas prácticas docentes de simulación frente al sistema escolar no constituyen, necesariamente, una acción de resistencia. Quienes afirman esto indican que esta oposición, profundamente conservadora, trasciende a los actores individuales, impregnando con su lógica y efectos a las instituciones en su conjunto.

(...) la escuela no es subversiva en ese sentido, es obediente ¿a qué? Al decreto ley, al dictamen de la provincial, aunque finalmente es casi una mentira porque no pasa nada de lo que supone que pasa, pero todos hacemos como que pasa. Hay que llenar el diagnóstico escolar, se llena el diagnóstico escolar; si hay que contratar una OTEC [Organismos Técnicos de Capacitación], se contrata ¿para qué? Porque hay que cumplir y se cumple. Entonces hay una lógica muy obediente en la escuela, los profesores en general son bastante obedientes. (FP-GI7).

En suma, al caracterizar las concepciones que los formadores tienen sobre el trabajo docente es relevante detenerse en la dualidad que construyen: por un lado, se define a los docentes a partir de una práctica heterónoma, que se ajusta a los requerimientos de la institución y del sistema escolar, sin dar espacio a recontextualizaciones y traducciones derivadas de una práctica autónoma; y, por otro, por desarrollar un comportamiento crítico, que reflexiona sobre sí mismos y la institución de la que forman parte, tomando decisiones sobre su desempeño de forma autónoma. En el espacio actual, marcado por el incremento de la presión sistémica producto de los dispositivos de rendición de cuentas, los formadores de profesores identifican en los docentes una conducta estratégica, de simulación pedagógica, que es interpretado por los formadores de profesores como resistencia pasiva o, por el contrario, como aquiescencia con el statu quo. 


\subsection{ESTAR EN LA ESCUELA: CONDICIONANTES Y POSIBILIDADES}

Un elemento común al conjunto de los formadores de profesores es la convicción de estar formando a docentes que trabajarán en instituciones escolares que no permiten el desarrollo y expansión de capacidades profesionales. Con diferentes matices, refieren los investigadores a un docente que aplica y no crea, con poca autonomía profesional, en condiciones laborales que antes que potenciar sus capacidades, las limitan:

Los profesores, durante mucho tiempo, -y ahí la política de la Concertación tuvo por supuesto responsabilidad-fueron pensados no como profesionales sino como técnicos, entonces de lo que se trataba [era que] implementaran los programas ministeriales, el currículum, algo exógeno a algo que ellos generan, a diferencia de lo que hace un profesor universitario (...) [que] desarrolla su temática de manera más autónoma, y eso yo creo, que junto con la pauperización de los ingresos, y por lo tanto de las personas que se formaban como técnicos educativos, yo creo que ha tenido un efecto súper grande, porque el profesor en general no se siente un profesional. (FP-GI7).

Esto implica la existencia de una distancia entre el discurso oficial sobre el docente y el concepto que tiene el formador de profesores al respecto. Así lo expresa uno de los formadores, quien recuerda sus comienzos como docente en un colegio:

(...) me hablaban de ser profesional, pero no tenía autonomía curricular, no tomaba decisiones curriculares. Entonces, del punto de vista de la profesionalidad docente, yo veía que ahí había mucho terreno que trabajar. (FP-GI5).

Por lo mismo, los formadores de profesores destacan que pese a caracterizar a los docentes como sujetos con capacidad reflexiva, esta cualidad aparece disminuida y dispersa en el contexto actual.

(...) el sujeto docente es reflexivo, lo que pasa es que en las condiciones actuales del trabajo docente, pocas son las ocasiones para sistematizar reflexividad, por eso es que hay que intencionarla. (FP2-GI2).

La subjetividad de los docentes se relaciona con condiciones de desempeño específicas, pero su voz no suele ser considerada en la construcción de discursos oficiales sobre su profesión, sus necesidades y carencias. Así lo expresa este formador de profesores:

[Existe la] necesidad de poner por sobre el tapete esta subjetividad docente que tiene que ver con situaciones laborales, los profesores tenemos necesidades sentidas que no siempre se ponen en juego y son otros los que nos dicen lo que necesitamos, y ahí emergen estas lecturas que los profesores necesitan mejorar el conocimiento de la disciplina, necesitan mejorar en la didáctica, pero ¿le han preguntado a los profesores qué necesitan realmente para desarrollar de buena manera su labor?. (FP-GI5).

En armonía con lo anterior, se enfatiza que el sujeto docente se encontraría en condiciones laborales marcadas por la precariedad, climas escolares deteriorados, relaciones laborales pobres y problemas de salud: 
(...) yo creo que los profesores tienen respecto de la escuela diferentes subjetividades. Creo que hay una subjetividad dolorosa que tiene que ver con el conjunto de relaciones, que muchas veces (...) son como parte de un círculo más vicioso, [donde] la calidad de vida no es buena (FP-GI7).

Al interior del discurso de los formadores de profesores es frecuente el uso de la categoría "malestar docente", en la misma línea que lo usó Esteve a finales de la década de 1980 (Martínez, Collazo y Liss, 2009) y Valdivia et al. (2003) para el caso chileno. Esto posibilita la construcción de una subjetividad conflictuada:

(...) también de la situación laboral de los profesores que emerge con mucha fuerza, muchas veces las discusiones que teníamos con los profesores, a todo esto de la subjetividad y de la reivindicación y del maltrato, del malestar, tenía que ver con situaciones de sobrevivencia, o sea los profesores que tenían problemas, las profesoras con su marido que no tenían una situación profesional y ellas eran profesionales, ellos no, habian conflictos familiares muchas veces porque la mujer tenía más recursos que el hombre, el hombre se sentía menos que la mujer, y eso es lo que yo vivía en las reuniones de profesores (...). (FP-GI5).

Este malestar, expresan, es potenciado por la constante vigilancia que sufren los docentes sobre su ejercicio profesional. En el espacio educativo esto se ha llevado a cabo a través de un complejo entramado de producción de un nuevo sujeto cultural, que considera la privatización, la estandarización, la examinación y el accountability (Carrasco, 2013). En lo concreto, esto se expresa en una subjetividad docente que si bien critica los objetivos de la actual política educativa, en la práctica internaliza sus procedimientos:

En mi escuela se ríen de todo lo que es la mejora, como que 'ya' asumen que están súper mal, pero agarran todo lo que es el control y la vigilancia y lo hacen carne, ellos nos van a venir a vigilar. Y todos los consejos [se dice] 'van a venir', 'van a venir', 'tenemos que estar preparados', y ellos lo transmiten. A los profesores de alguna u otra manera igual les llega eso. (FP-GI6).

Varios de los formadores de profesores coinciden en que los docentes no cuentan con una "voz" que exprese lo que sienten o necesitan, y tampoco para abordar aspectos más generales del ejercicio de la profesión. En ese contexto surgen ideas que refieren al silenciamiento:

(...) es un sujeto que ha sido silenciado. O sea, si no lo miramos en abstracto, sino que miramos la situación de los profesores en Chile, que la más de las veces ha sido acallado y también autosilenciado, que ha sido subordinado, autosubordinado a los discursos poderosos. (FP-GI2).

Esta debilidad de la voz docente en el espacio público deriva de las transformaciones producidas en las condiciones laborales de desempeño docente, la desvalorización del saber docente frente a otros saberes y, por último, el debilitamiento como actor colectivo. En conjunto, esto explicaría su dificultad para participar de los debates pedagógicos, pero también en aquellos de política educativa y en lo atingente a su propia profesión.

\section{DISCUSIÓN Y CONCLUSIÓN}

Las concepciones que los formadores de profesores tienen sobre los saberes y la subjetividad docente se mueven en una tensión entre dos enfoques: uno de tipo racionalista, 
marcado por la búsqueda de la eficacia; y otro de tipo antropológico y fenomenológico, que pone su mirada en los procesos de desarrollo del sujeto (Malet, 1998). Más próximos al segundo tipo, los entrevistados en esta investigación conciben el saber docente como uno de carácter situado, biográfico, que se despliega en la acción, que se construye dialógicamente y reside en el colectivo. Este saber, construido en la práctica y en la cultura escolar, constituye un acervo de conocimiento práctico para los docentes, en gran medida formado inductiva e intuitivamente, que se consolida en la experiencia de estar y ser parte de la institución escolar. En palabras de un formador, este "saber moverse" en la escuela complementa el conjunto de saberes aportados por la formación inicial en la educación superior. En suma, las concepciones de los formadores de profesores en estudio se acercan a una noción de profesionalización entendida como un proceso permanente, mediante el cual se adquiere y construye identidad y conocimientos como resultado de un "devenir" (Malet, 1998). Es un proceso de "llegar a ser" que no acaba, porque está siempre como un acto presente, como una "reconstrucción" (Connelly y Clandinin, 1995).

Cercano a la noción de sabiduría de la práctica, esta concepción del saber docente da cuenta de lógicas que trascienden lo esperable por las políticas públicas en las que prima una concepción de la formación docente como el resultado de una "inyección" de saberes necesarios para ejercer en la enseñanza. En el presente, el despliegue y la expansión de los saberes docentes aparecen condicionados por el predominio del modelo de "docencia efectiva". Este modelo se traduce en la promoción de políticas y prácticas estipuladas como deseables dentro de las instituciones escolares, que buscan configurar un modo determinado de "ser docente". Al respecto, Shulman (2005) señala que en algunas investigaciones sobre docencia efectiva se han generado índices que la política ha transferido un tanto acríticamente a estándares de desempeño. Así, sólo aquellos ítems que resultan medibles a través de estándares serían considerados pertinentes en la configuración del saber del profesor. Frente a esto, el diagnóstico de los formadores de profesores implicados en el presente proyecto es radicalmente crítico. Para estos no es posible reducir el saber docente sólo a contenidos medibles, existiendo un cúmulo de experiencias y saberes docentes que quedan fuera de la mirada de las políticas educativas.

Desde su perspectiva, existe una tensión entre la concepción poliédrica del saber docente que manifiestan los formadores de esta institución de educación superior y la noción restringida que existiría en las políticas sobre la docencia. A partir de reconocer este hiato, surge la necesidad de conocer cuáles son los saberes que distinguen a la profesión docente, muchos de los cuales no son considerados por las evaluaciones estandarizadas. En su manifestación más propia, y en la mirada sostenida por los formadores de profesores de este proyecto, este es un saber que se fundamenta en el ejercicio reflexivo-crítico de un sujeto que construye conocimiento y que despliega y avanza en la comprensión de la complejidad de la realidad que su praxis abarca, un conocimiento que se "sitúa en un marco donde las emociones, sensaciones, vivencias, experiencias, tienen un papel fundamental (...)" (FP-GI5).

La explicitación de una mirada distinta e interpeladora que emerge de los formadores de formadores en torno al lugar que ocupa el docente y su saber en el contexto y escenario de la política pública nacional no implica per se su transformación, sino que su potencia está en que, a partir de dicha explicitación, se puede pensar que la transformación puede acontecer en otro lugar, en el espacio formativo que pone en relación de diálogo a futuros docentes con sus formadores. Desde la óptica de los formadores de profesores, un punto de 
convergencia relevante en este proceso es la concepción del docente como sujeto histórico, en tanto condicionado por las coordenadas tiempo y espacio en las que vive, pero pudiendo trascenderlas con su acción profesional.

Si bien la ausencia de la historicidad de los docentes en las políticas tiende a reproducirse en el ámbito del saber académico, el grupo de formadores entrevistado no solo reconoció su existencia, sino que para muchos constituye la base real del trabajo académico-pedagógico del docente. Estos saberes docentes auto-generados en la escuela son interpretados por muchos autores como la historicidad propia de vivir la escuela como un ser social, que es producido por políticas, pero que a la vez produce una realidad educativa nueva. Goodson (2003) llama a este proceso "memoria corporativa", en tanto cadena de transmisión identitaria desde el vocacionalismo del "viejo profesional' al "nuevo profesional", mientras que Giroux (2003) lo denomina el desarrollo de una conciencia histórica crítica, y Freire (1994) como la construcción de un sujeto histórico, desde el potencial que cada sujeto tiene de trascender la dominación; es decir, pueden ser modos históricos de subjetivación para producir subjetividad (Fernández, 2011).

Estudios relacionados con la voz y subjetividad de los docentes concluyen que las resistencias de los docentes de aula a participar de actividades impulsadas por profesionales que vienen desde fuera del colegio, se producen precisamente por la falta de reconocimiento del saber docente por parte de los saberes externos a los establecimientos, no incorporando sus tiempos y espacios constitutivos, limitando su autonomía e incidencia en las políticas escolares (Guerrero, 2005). En ello consiste precisamente uno de los desafíos de la FID, cuando la tendencia hegemónica es a desprenderse de las líneas curriculares que promueven la historicidad de los sujetos y, por lo tanto, del reconocimiento a su condición de sujeto político colectivo.

El análisis de los datos permite comprender que existen matices en el planteamiento de las posibilidades de salida a las condicionantes del trabajo docente. Las hay más pesimistas, como aquellos formadores de profesores que se sitúan desde sus investigaciones con respuestas más pasivas ("acomodaciones", "adaptación", "resistencias tímidas y/o individuales", entre otras), mientras que otro grupo se sitúa en una apuesta más activa de resistencia ("organizaciones", "colectivos" y "sindicatos docentes"). Ambos tipos de resistencia expresan saberes propios del trabajo docente, por lo que las instituciones formadoras de profesores tienen el desafío de reconocer esta multiplicidad de saberes existentes, trabajando con ellos, potenciándolos y convirtiéndolos en una herramienta para poner en marcha la autonomía docente.

A partir de la presente publicación se ha esbozado una primera mirada a la interrogante sobre cuáles son las concepciones que los formadores de profesores poseen con respecto a los saberes y subjetividad de los profesores del sistema escolar chileno. La respuesta a esta interrogante puede ser rotunda en el sentido de la profundidad de lo analizado, pero a la vez acotada, pues las categorías y su jerarquización simbólica está supeditada al contexto situado de la experiencia y visión de los actores objeto de estudio.

Uno de los aspectos centrales del estudio lo constituye la discusión de las investigaciones presentes en el marco referencial y teórico. Si bien la conceptualización asumida y el análisis de los enfoques en uso sobre la subjetividad docente y las precisiones teóricas sobre las concepciones y del formador de profesores dan cuenta de la multidimensionalidad del fenómeno, cada una de ellas ofrece un aspecto parcial y a veces contradictorio del objeto de estudio. 
Estudios Pedagógicos, vol. XL, Número Especial 1: 183-203, 2014

SUBJETIVIDADES Y SABERES DOCENTES EN EL SISTEMA EDUCATIVO CHILENO: UN ANÁLISIS DESDE LAS CONCEPCIONES DE FORMADORES DE PROFESORES

De este modo, el escrito responde al propósito planteado, puesto que analiza las concepciones de los sujetos informantes, confirmando el escenario complejo en que se desarrolla la formación docente, tanto desde el punto de vista personal como sistémico.

Entre las limitaciones que se pueden asumir se encuentra no haber considerado la voz de los propios docentes visibilizados en las concepciones de los formadores de profesores, haber incorporado una muestra más amplia de informantes clave o haber desarrollado un estudio que incorpore la docencia o práctica de los formadores de profesores para contrastar las concepciones esbozadas. Sin duda, son muchas las interrogantes que se pueden extraer de este estudio, una de ellas es preguntarse por el rol de la formación pedagógica/no pedagógica de los formadores de profesores o el papel de la edad, experiencia, formación permanente y teoría en la trasformación de la formación de profesores, entre otras. Todas ellas constituyen potenciales líneas de investigación para futuros estudios en el área.

En síntesis, queda en evidencia que las concepciones de los formadores de profesores presentan un entramado de ideas, prácticas, expectativas, nudos críticos y esperanzas de cambio que justifican su estudio, instando a profundizar en nuevas investigaciones que permitan generar una base científica que alimente la toma de decisiones respecto a la valorización de la formación de profesores en Chile.

\section{REFERENCIAS BIBLIOGRÁFICAS}

Acanda, L. (2008). La problemática del sujeto y los desafíos para la teoría de la educación. Recuperado desde http://www.correntroig.org/IMG/pdf/La_problematica_del_sujeto.pdf

Acuña, F.; Assaél, J.; Contreras, P. y Peralta, B. (2014). La traducción de los discursos de la política educativa en la cotidianeidad de dos escuelas municipales chilenas: La metáfora médica como vía de análisis. Psicoperspectivas, vol.13, n.1, 46-55.

Alvarado, L. (2006). Formação Continuada de Professores em Serviço: Formação de Formadores. In M. Monteiro y M. Muller, Profissionais da Educação: políticas, formação e pesquisa. Cuiabá: EdUFMT.

Arévalo, A. (2010). La experiencia de sí como investigadora. En J. Contreras y N. Pérez de Lara (Comps.), Investigar la experiencia educativa (pp. 188-198). Madrid: Morata.

Avalos, B. (2005). La formación de profesores y su desarrollo profesional. Prácticas innovadoras en busca de políticas. El caso de Chile. En C. Cox (Ed.), Políticas educacionales en el cambio de siglo (pp. 559-594). Santiago: Editorial Universitaria.

Bauman, Z. (2000). Trabajo, consumismo y nuevos pobres. Barcelona: Gedisa.

Becerra, S. (2009). Prejuicio étnico docente: los efectos psicosociales en el alumno mapuche y su reproducción en las prácticas de convivencia educativa en establecimientos de alta vulnerabilidad social. Informe final FONDECYT 11090021.

Blanco, M. (2012). Autoetnografía: una forma narrativa de generación de conocimientos. Andamios. Revista de Investigación Social, vol.9, n.19, 49-74.

Bullough, R., \& Pinnegar, S. (2001). Guidelines for Quality in Autobiographical Forms of SelfStudy Research. Educational Researcher, vol.30, n.3, 13-21.

Carrasco, A. (2013). Mecanismos performativos de la institucionalidad educativa en Chile: pasos hacia un nuevo sujeto cultural. Observatorio Cultural, n.15, 4-10.

Castillo, E. (2006). La formación continua de profesores con universidades chilenas. Una alianza que dinamiza el sistema educativo. Santiago: CPEIP.

Cochran-Smith, M., \& Fries, K. (2005). The AERA panel on research and teacher education: context and goal. In M. Cochran-Smith, \& K.M. Zeichner (Eds.), Studying teacher education: The 
Estudios Pedagógicos, vol. XL, Número Especial 1: 183-203, 2014 SUBJETIVIDADES Y SABERES DOCENTES EN EL SISTEMA EDUCATIVO CHILENO: UN ANÁLISIS DESDE LAS CONCEPCIONES DE FORMADORES DE PROFESORES

report of the AERA Panel on Research and Teacher Education (pp. 69-110). Washington, DC: American Educational Research Association and Mahwah, NJ. Lawrence Erlbaum Associates, Publishers.

Connelly, M. y Clandinin, J. (1995). Relatos de experiencia e investigación narrativa. En J. Larrosa, R. Arnaus, V. Ferrer, N. Pérez de Lara, M. Connelly, J. Clandinin y M. Greene. Déjame que te cuente. Ensayos sobre narrativa y educación (pp. 11-59). Barcelona: Laertes Educación.

Consejo Asesor Presidencial para la Calidad de la Educación. (2006). Informe final. Última visita 05 octubre de 2014. Recuperado desde http://www.facso.uchile.cl/psicologia/epe/_documentos/ GT_cultura_escolar_politica_educativa/recursos\%20bibliograficos/articulos\%20relacionados/ consejoasesorpresidencialparalacalidaddelaeducacion(2006)informefinal.pdf

Cornejo, R. (2009). Condiciones de trabajo y bienestar/malestar docente en profesores de enseñanza media de Santiago de Chile. Educación y Sociedad, vol.30, n.107, 409-426.

Cruz, M., Reyes, M. y Cornejo, M. (2012). Conocimiento Situado y el Problema de la Subjetividad del Investigador/a. Cinta de Moebio, vol. 45, 253-274.

Darlin-Hammond, L., Chung Wei, R., \& Andree, A. (2010). Professional Learning in the Learning Profession: A Status Report on Teacher Development in the United States and Abroad. USA: National Staff Development Council.

Day, C. (2006). Pasión por enseñar: la identidad personal y profesional del docente y sus valores. Madrid: Narcea.

Day, Ch., \& Guy, Q. (2010). The New Lives of Teachers. Londres: Routledge.

El Plan Maestro. (2014). Qué es el Plan Maestro. Qué hacemos y cómo trabajamos. Última visita 02 de septiembre de 2014. Recuperado desde: http://www.elplanmaestro.cl/que-es-plan-maestro/

Fernández, A. (2011). Política y subjetividad. Asambleas barriales y fábricas recuperadas (3 ed.). Buenos Aires: Biblos.

Freire, P. (1994). Educación y participación comunitaria. En M. Castells, R. Flecha, P. Freire, H. Giroux, D. Macebo y P. Willis. Nuevas perspectivas críticas en educación (pp. 83-96). Barcelona: Paidós.

García, S. (2003). Fertilizaciones cruzadas entre la psicología social de la ciencia y los estudios feministas de la ciencia. Athenea Digital, vol.4, 109-150.

Giroux, H. (2003). Pedagogía y política de la esperanza: Teoría, cultura y enseñanza. Buenos Aires: Amorrortu.

Gómez, V. (2012). Creencias epistemológicas, sobre el aprendizaje y la enseñanza en contextos de pobreza, en estudiantes de pedagogía y formadores de profesores. Informe final FONDECYT, 1120550.

González Rey, F. (2002). Sujeto y subjetividad. Una aproximación histórico cultural. México: Thompson.

Goodson, I. (2003). Hacia un desarrollo de las historias personales y profesionales de los docentes. Revista Mexicana de Investigación Educativa, vol.8, n.19, 733-758.

Guerrero, P. (2005). Estudio de las resistencias de los profesores a una estrategia para el desarrollo de la creatividad en tres unidades educativas. Psykhe, vol.14, n.1, 31-45.

Harding, S. (1996). Ciencia y feminismo. Madrid: Morata.

Haynes, K. (2006). Linking narrative and identity construction: using autobiography in accounting research. Critical Perspectives on Accounting, vol.17, n.4, 399-418.

Jackson, P. (1990). Vida en las aulas. Madrid: Morata.

Joram, E. (2007). Clashing epistemologies: Aspiring teachers', practicing teachers', and professors' beliefs about knowledge and research in education. Teaching and Teacher Education, vol.23, n.2, 123-135.

Korthagen, F. (2004). In search of the essence of a good teacher: Towards a more holistic approach in teacher education. Teaching and Teacher Education, vol.20, n.1, 77-97.

Koster, B. (2005). Quality requirements for teacher educators. Teaching an Teacher Education, vol.21, n.2, 157-176.

LEY No 20.370. (2009). Ley General De Educación. Santiago: Diario Oficial de la República de 
Estudios Pedagógicos, vol. XL, Número Especial 1: 183-203, 2014

SUBJETIVIDADES Y SABERES DOCENTES EN EL SISTEMA EDUCATIVO CHILENO: UN ANÁLISIS DESDE LAS CONCEPCIONES DE FORMADORES DE PROFESORES

Chile 12 de septiembre de 2009.

López de Maturana, S. (2005). La construcción sociocultural de la profesionalidad docente: compromiso social, político y pedagógico. Informe FONDECYT 1050621.

Loughran, J., Hamilton, M.L., Kubler, V., \& Rusell, T. (2007). International Handbook of SelfStudy of Teaching and Teacher Education Practices. Netherlands: Springer.

Malet, R. (1998). L'identité en formation. Phénoménologie du devenir enseignant. Paris: L'Harmattan.

Mancebo, D. (2010). Subjetividade docente. En D. Oliveira, A. Duarte y L. Vieira (Orgs.), Dicionário: trabalho, profissão e condição docente. Belo Horizonte: UFMG. Faculdade de Educação. [Versión CDROM].

Martínez, D., Collazo, M. y Liss, M. (2009). Dimensiones del trabajo docente: una propuesta de abordaje del malestar y el sufrimiento psíquico de los docentes en la Argentina. Educación y Sociedad, vol.30, n.107, 389-408.

MINEDUC. (2005). Informe Comisión sobre formación inicial docente. Santiago de Chile: Dirección de comunicaciones. Serie Bicentenario.

MINEDUC. (2014). Evaluación Inicia. Antecedentes de las Pruebas Inicia. Última visita 05 noviembre de 2014. Recuperado desde http://www.mineduc.cl/index2.php?id_portal=79\&id_ seccion $=4243 \&$ id_contenido $=20558$

Miranda, C. (2004). Formación permanente de profesores. Impacto en sus competencias profesionales. Santiago: Pontificia Universidad Católica de Chile.

Montecinos, C. (2007). El impacto de la formación práctica en el proceso de aprender a enseñar. Informe final FONDECYT 1070807.

Moreno, M. y Azcárate, C. (2003). Concepciones y creencias de los profesores universitarios de matemáticas acerca de la enseñanza de las educaciones diferenciales. Enseñanza de las Ciencias, vol.21, n.2, 265-280.

Núñez, I. (2003). El profesorado, su gremio y la reforma de los noventa: presiones de cambio y evolución en la cultura docente. En C. Cox (Ed.), Políticas educacionales en el cambio de siglo (pp. 455-517). Santiago: Editorial Universitaria.

Organización para la Cooperación y el Desarrollo Económicos. (2004). Revisión de Políticas Nacionales de Educación: Chile. París: OCDE.

Organización para la Cooperación y el Desarrollo Económicos. (2009). La educación superior en Chile. Santiago: OCDE-Banco Internacional para la Reconstrucción y el Desarrollo / Banco Mundial.

Pozo, J., Scheuer, N., Pérez, M., Mateos, M. y Monserrat, M. (2006). Nuevas formas de pensar la enseñanza y el aprendizaje. Las concepciones de profesores y alumnos. Barcelona: Grao.

Reyes, L., Cornejo, R., Arévalo, A. y Sánchez, R. (2010). Ser docente y subjetividad histórica en el Chile actual: discursos, prácticas y resistencias. Polis, Revista de la Universidad Bolivariana, vol.9, n.27, 269-292.

Reyes, L., Toledo, M. y Egaña, L. (2013). École, communauté et sujet enseignant. Le projet historique de la 'Escuela Consolidada Miguel Dávila Carson'. Chili, 1953-1977. Paedagogica Historica: International Journal of the History of Education, vol.49, n.2, 425-443.

Rivera, P. (2014). Desarrollo profesional docente desde la voz del formador de formadores. Tesis Doctoral. Universidad Austral de Chile: Valdivia.

Rockwell, E. (2009). La experiencia etnográfica. Historia y cultura en los procesos educativos. Buenos Aires: Paidós.

Russell, T., McPherson, S., \& Martin, A. (2001). Coherence and Collaboration in Teacher Education Reform. Canadian Journal of Education, vol.26, n.1, 37-55.

Sánchez, E. (2012). Concepciones de la formación de profesores de ciencias en estudiantes de segundo ciclo básico. Tesis de Grado: Universidad Austral de Chile: Valdivia.

Shulman, L. (2005). Conocimiento y enseñanza: Fundamentos de la nueva reforma. Profesorado. 
Revista de currículum y formación del profesorado, vol.9, n.2, 1-30.

Sisto, V. (2012). Identidades Desafiadas: Individualización, Managerialismo y Trabajo Docente en el Chile Actual. Psykhe, vol.21, n.2, 35-46.

Strauss, A. y Corbin, J. (2002). Bases de la investigación cualitativa. Técnicas y procedimientos para desarrollar la teoría fundamentada. Antioquía, Colombia: Universidad de Antioquía.

Tardif, J. (2006). L'évaluation des compétences. Montréal: Ca.Chenelière Éducation.

Taylor, P. (2006). The choreography of accountability. Journal Education Policy, vol.21, n.2, 201-214.

Theobald, N. (1990). An examination of the personal, professional, and school district characteristics on public school retention. Economics of Education Review, vol.9, n.3, 241-250.

UNESCO. (2005). Condiciones de trabajo y salud docente. Estudios de casos en Argentina, Chile, Ecuador, México, Perú y Uruguay. Santiago: UNESCO-OREALC.

Valdivia, G., Avendaño, C., Bastías, G., Milicic, N., Morales, A. y Scharager, J. (2003). Estudio de la salud laboral de los profesores en Chile. Santiago: Pontificia Universidad Católica de Chile.

Van Manen, M. (2003). Investigación educativa y experiencia vivida. Barcelona: Idea Books.

Wittrock, M. (1986). Handbook of Research on Teaching. New York: Macmillan.

Wright, C. (1969). La imaginación sociológica. México: Fondo de Cultura Económica.

Zeichner, K. (2004). New Visions of Teacher Professional Development. Yearbook of the National Society for the Study of Education, vol.103, n.1, 180-227.

Zemelman, H. (1997). Sujetos y subjetividad en la construcción metodológica. En E. León y H. Zemelman (Eds.), Subjetividad: umbrales del pensamiento social (pp. 21-35). Barcelona: Antrophos.

Zemelman, H. (2010). Sujeto y Subjetividad: la problemática de las alternativas como construcción posible. Polis, Revista de la Universidad Bolivariana, vol.9, n.27, 355-366. 
